\title{
Biomarkers of Traumatic Brain Injury in the Geriatric Population
}

\author{
Darian Rice • Kevin K. W. Wang • Steven Robicsek
}

Published online: 18 July 2012

(C) Springer Science+Business Media, LLC 2012

\begin{abstract}
Traumatic brain injury (TBI) remains a significant cause of morbidity and mortality, and advanced age is an independent predictor of worse outcome. Mortality is nearly double that of younger TBI patients, regardless of injury severity. In addition, geriatric patients are more prone to complications, have longer hospital stays, and require long-term rehabilitative services, the cost of which can be a significant burden. These observations are often attributed to anatomic and physiologic changes that occur with age, as well as patient comorbidities and the medications required to treat those conditions. Although current diagnostic and treatment guidelines have led to improved outcomes in the general population, data regarding the specific management of geriatric patients remain sparse. The identification and management of TBI has been the focus of both basic science and clinical investigations. Recent advances in molecular biology have identified specific markers of brain cell damage that may offer insight to the nature and progression of injury, lead to improved monitoring and diagnostic capability, and potentially guide therapy. This review highlights some of the latest technology that may pave the way for advanced diagnostic and treatment modalities.
\end{abstract}

\section{Rice}

Department of Anesthesiology, Naval Medical Center Portsmouth,

620 John Paul Jones Circle,

Portsmouth, VA 23708-2197, USA

K. K. W. Wang

Departments of Psychiatry and Neuroscience,

University of Florida,

Gainesville, FL, USA

S. Robicsek $(\square)$

Department of Anesthesiology,

University of Florida College of Medicine,

1600 Southwest Archer Road,

Gainesville, FL 32610-0254, USA

e-mail: robicsek@ufl.edu
Keywords Traumatic brain injury $\cdot$ Geriatric . Biomarker · Age

\section{Introduction}

On January 1, 2011, as the baby boomers begin to celebrate their 65th birthdays, 10,000 people will turn 65 every day-this will continue for 20 years.

- Alliance for Aging Research [1]

Traumatic brain injury (TBI) remains a significant cause of morbidity and mortality in people of all ages, affecting nearly 1.4 million patients annually in the United States alone. About 235,000 of these patients will require hospital admission, and $20 \%$ will likely succumb to their injuries [2]. Advanced age, considered those over the age of 65 years, is an independent predictor of poorer outcomes [3]. In 2003, Hukkelhoven and colleagues [4] analyzed 5,600 patients with TBI, which identified an improved method of predicting outcome. They noted a nearly linear association between age and poor outcome and concluded that with each 10-year increment in age, outcome worsens by $40 \%-50 \%$, and is independent of other risk factors. And according to the U.S Census Bureau, the geriatric demographic will continue to expand both in absolute numbers as well as percent of the total population (Fig. 1), with the percentage of the U.S. population increasing from $13 \%$ in 2010 to over $22 \%$ by 2050 [5].

Among geriatric patients, TBI represents approximately 80,000 annual emergency department visits, of which about $75 \%$ will require hospitalization [6]. Mortality rates are nearly double that of their younger counterparts, for even mild degrees of head injury [7]. In addition, the number of these patients who suffer long-term psychological and physical disability remains high, and the monetary burden is 


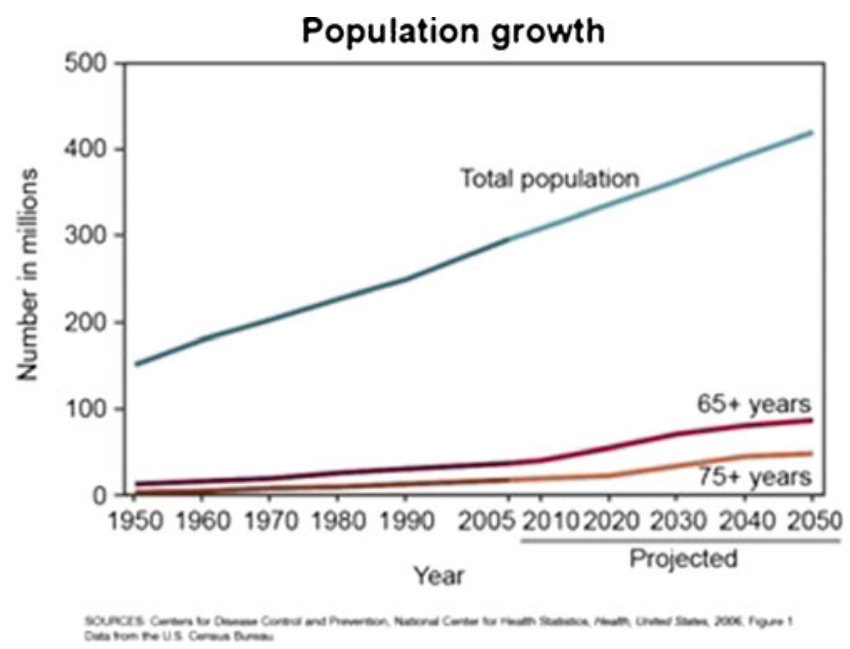

Fig. 1 United State population growth projection. Source: U.S Census Bureau

significant [6]. When overall recovery and outcome were assessed by the Glasgow Outcome Score (GOS), the proportion of survivors in the category of "good recovery" all declined with increasing age [8]. Efforts to identify strategies to optimize the care of these patients and improve outcomes thus far have not yielded promising results. However, recent advances in molecular biology and proteomics have identified potential biochemical markers of neuronal injury specific to TBI. Such technology has the potential improve diagnostic and prognostic capability, as well as serve as a guide to clinical management [9॰].

\section{Epidemiology of TBI}

TBI has a bimodal age distribution, with the first peak at 1519 years and the second appearing as age exceeds 65 years. The primary causes of TBI include motor vehicle crashes (MVC), falls, and violent crime. In younger adults, the primary cause is MVC, whereas in older patients falls are more prevalent.[10] Interestingly, the most common type of fall is from standing height, generally considered to be a low-energy impact [11•]. The factors that increase the susceptibility of elderly patients to injury and delay recovery are multifactorial. Issues specific to the geriatric population include anatomic and physiologic changes that occur with increasing age, as well as comorbidities and the medications required to treat those conditions [10]. Alcohol intake, suicide, and lack of protective equipment or appropriate preventive measures also have been implicated $[6,11 \bullet]$. Patients with fall-related TBI tend to have more comorbidities than those injured in MVCs [6]. The types of comorbid conditions between the two mechanisms of injury that are similar include cardiovascular and cerebrovascular disease, hypertension, cardiac arrhythmias, diabetes mellitus, and fluid and electrolyte imbalances; whereas the incidence of dementia, depression, and Parkinson's disease is significantly increased in fall-related injuries [6]. In addition, patients with pre-existing dementia or who have underlying cognitive impairment are not only at higher risk for falls, but also require longer recovery time [6].

\section{Pathophysiology of Traumatic Brain Injury}

In TBI, the primary injury may be focal or poorly defined with various coup and contra-coup involvement. Regardless of mechanism, head injuries result in a spectrum of clinical manifestations ranging from confusion and memory loss to paralysis and irreversible coma. Focal lesions, often seen on head computed tomography (CT), have the potential to expand and mechanically compress brain parenchyma, leading to detrimental shift, herniation, or brainstem compression. Equally devastating are the poorly defined white matter neuronal injuries, now referred to as diffuse axonal injury (DAI) $[12,13]$. In the elderly, age-related changes and comorbid conditions tend to worsen the extent of these injuries, complicate clinical management, and slow recovery. Confounding factors frequently seen in the elderly include cerebral atrophy and dural adhesion that may alter the intracranial architecture and, therefore, injury progression. In addition, pre-existing diseases such as hypertension, cerebrovascular atherosclerosis, and small vessel disease (SVD) are known to cause a spectrum of vascular derangements. Deficits commonly seen include subclinical ischemic or hemorrhagic lesions such as lacunar infarcts and microbleeds, respectively, to overt cerebrovascular accident (CVA). Not surprising, patients on anticoagulant therapy for arrhythmias and/or vascular disease are at risk for increased injury severity, resulting in higher morbidity and mortality [6].

Upon impact, hemorrhagic lesions lead to hematoma formation and the ensuing inflammatory response triggers edema accumulation. Hematoma can take many forms depending on location; epidural, subdural, and/or subarachnoid. A common consequence of hematoma accumulation and edema is increased intracranial pressure (ICP) that can ultimately attenuate cerebral blood flow $(\mathrm{CBF})$. During periods of increased ICP, cerebrospinal fluid (CSF)-containing structures such as the ventricles will be compressed, thereby causing a downward displacement of CSF toward the spinal cord. Asymmetric expansion of intracranial contents, such as occurs with an expanding hematoma, also may cause the brain parenchyma to herniate through semi-rigid structures such as the falx cerebri, tentorium cerebelli, and foramen magnum. With increasing ICP, cerebral venous outflow also may be impaired, followed by arterial inflow. Obstruction to venous outflow results 
in increased ICP as venous volume expands, whereas impedance to arterial inflow reduces cerebral blood flow, both of which reduce cerebral perfusion pressure (CPP) [14].

TBI is not static in nature, but rather continues to evolve over the subsequent hours to days. The extent of injury is not limited to the initial gross structural derangement, but progresses and becomes amplified as secondary injury leads to tissue ischemia, inflammation, oxidative stress, and potentially neuronal cell death. This can be seen histologically as the ischemic, or traumatic penumbra, the functionally impaired region immediately adjacent to the primary injury that remains at risk but may be recoverable. Damage following TBI has been reviewed in detail by Maas et al. [15]. On the macroscopic level, injury includes shearing of whitematter tracts, focal contusions, vascular tears, and hematoma accumulation. At the cellular level, early neurotrauma events (which can occur minutes to hours after initial injury) include microporation of membranes, leaky ion channels, and stearic conformational changes in protein structure [15]. Neuroinflammation is also a predominant feature of secondary injury [16]. Pitts and McIntosh [17] provided a more detailed explanation for these findings and coined the term "tertiary injury" to describe the intricate molecular and cellular alterations that contribute to these findings. These alterations include loss of cell membrane architecture, ion channel integrity, and proper regulation of intracellular metabolic and synthetic mechanisms $[12,17]$. Cellular repair mechanisms are energydependent and ischemia leads to mitochondrial dysfunction and power failure as adenosine triphosphate (ATP) production is drastically reduced. This accelerates oxidative stress due to impaired free radical clearance, which further impedes recovery. In older adults, the inability of cells to efficiently clear free radicals has been previously implicated in the natural aging process, which may significantly increase the injury burden among elderly patients [18]. If unresolved, the terminal event is the activation of neuronal necrotic and apoptotic pathways, and subsequent cellular demise (Fig. 2). At the molecular level, these pathways are initiated by elevated neuronal calcium levels present post-injury via calcium-dependent enzyme activation. Known targets of these enzymes include caspase- 3 and calpain activation, known to be central players in cellular apoptosis and necrosis, respectively.

\section{The Role of Biochemical Markers in TBI}

TBI is a heterogeneous disorder with different forms of presentation. The unifying factor is that brain damage results from external forces as a consequence of direct impact, rapid acceleration or deceleration, a penetrating object (eg, gunshot), or blast waves from an explosion. The nature, intensity, direction, and duration of these forces determine the pattern and extent of damage. While little can be done to undo the primary insult, treatment is centered on the prevention and management of secondary and tertiary injuries. Maintaining cerebral homeostasis in the face of TBI is crucial. Optimizing cerebral perfusion by supporting mean arterial pressure (MAP) while minimizing ICP and central metabolic rate of oxygen $\left(\mathrm{CMRO}_{2}\right)$ remain the central tenets of physiologic management following TBI. However, while these clinical measurements offer some global information regarding the state of the brain, they provide very little data on injury progression or recovery.

The search for specific markers of brain injury has included many potential targets. Clinically, potentially accessible sources of these biological markers include CSF, cerebral interstitial fluid (usually obtained experimentally by microdialysis), and serum. Particularly useful biomarkers would be those released in a temporal manner as primary, secondary, and tertiary injuries evolve. On the macroscopic level, impaired perfusion due to vascular disruption or inflammation may lead to regional or global cerebral ischemia. As a consequence, cellular dysfunction ensues leading to impaired gene expression and protein synthesis, and the appearance of markers of cell stress. Genomic indicators reflecting synthetic cellular functions would be unpredictable and therefore unreliable indicators of injury progression. However, direct cellular injury leads to the release of neuronal debris into the extracellular space and CSF, and thus has the potential to serve as a specific biochemical marker of TBI. In addition, markers of intracellular molecular cascades upregulated in the face of cellular inflammation and repair also may be sensitive indicators [19]. To date, a number of neuro-specific markers, primarily proteins and peptide fragments, have been identified that could provide information on proteolytic mechanisms of TBI (necrotic oncosis vs apoptosis), cell type, and subcellular localization (eg, axon vs cell body and synapse) of injury (Table 1). Both quantitative and qualitative assessment of neuronal injury have been suggested using panels of the multiple biomarkers. Candidates thus far include neural cell body injury markers such as neuron-specific enolase (NSE,) and ubiquitin C-terminal hyrdrolase-1 (UCH-L1) [9•, 39, 40••]; gliosis and/or glial injury markers $\mathrm{S} 100 \beta$ (calciumbinding protein localized to glial cells) and glial fibrillary acidic protein (GFAP) [33, 34, 41••]; the demyelination indicator myelin basic protein (MBP) [20]; indicators of axonal injury such as axonal microtubule associated protein (Tau, or cleaved Tau) [35] and neurofilament protein- $\mathrm{H}$ and $-\mathrm{L}$ (NF-H) [36, 37, 39]; dendritic injury marker microtubule associated 
Fig. 2 Conceptual diagram illustrating the intracellular derangements in TBI

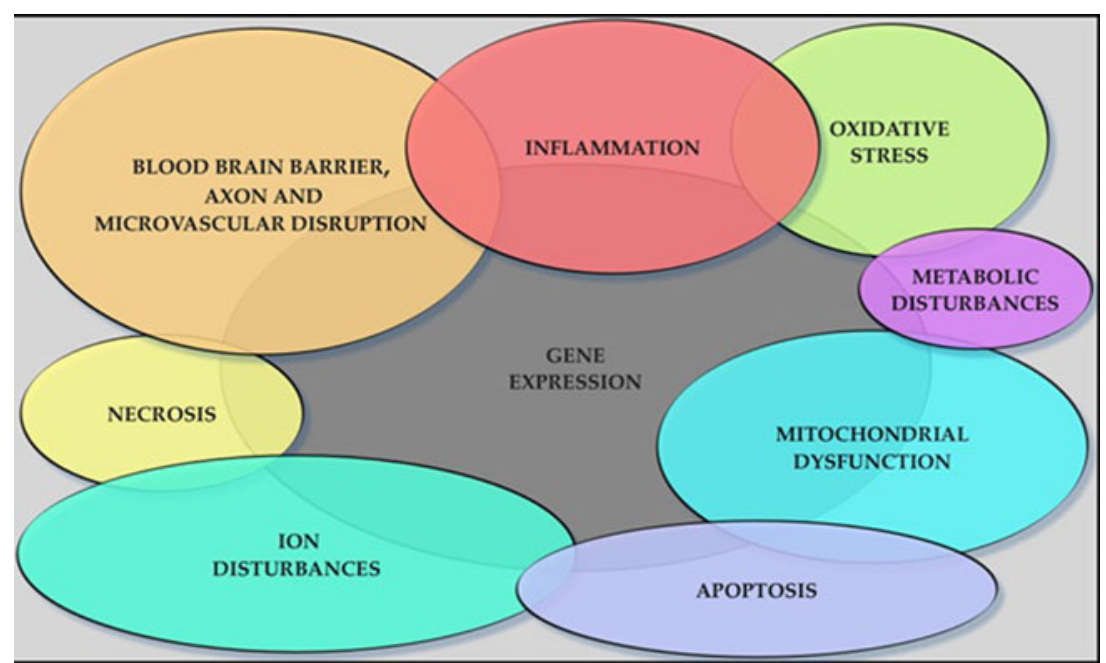

protein-2 (MAP-2) [38]; and a number of proinflammatory cytokines such as interleukins 1, 6, and 8 (IL-1, IL-6, IL-8), tumor necrosis factor alpha $(\mathrm{TNF} \alpha)$, and interferon gamma $($ IFN- $\gamma)[42,43]$. AlphaII-spectrin, a major substrate for both calpain and caspase-3 is also a promising marker [19, 39]. Its breakdown products include spectrin breakdown product (SBDP)-150 and SBDP-145 from calpain activation (primary during acute necrotic neuronal injury or death) and SBDP-120 from caspase- 3 proteolysis seen during the delayed apoptosis stage [21, 22]. Because AlphaII-spectrin is in mainly in axons, they are also considered axonal injury indicators. $15-\mathrm{kDa}$ cytoplasmic fatty acid-binding proteins (FABPs) and

Table 1 Representative potential biomarkers of neuronal injury

\begin{tabular}{|c|c|}
\hline \multirow[t]{2}{*}{$\begin{array}{l}\text { Neuronal injury and } \\
\text { necrosis/apoptosis }\end{array}$} & $\begin{array}{l}\text { SBDP-150 and SBDP-145 } \\
\text { (calpain mediated acute neural necrosis) }\end{array}$ \\
\hline & $\begin{array}{l}\text { SBDP-120 (caspase mediated } \\
\text { delayed neural apoptosis) }\end{array}$ \\
\hline Neural cell body injury & $\begin{array}{l}\text { Ubiquitin C-terminal hyrdrolase-1 } \\
\text { (UCH-L1); Neuron-specific enolase } \\
\text { (NSE) }\end{array}$ \\
\hline \multirow[t]{2}{*}{ Glial Injury or Gliosis } & S100 $\beta$ \\
\hline & Glial fibrillary acidic protein (GFAP) \\
\hline $\begin{array}{l}\text { Indicators of } \\
\text { demyelination }\end{array}$ & Myelin basic protein (MBP) \\
\hline \multirow[t]{2}{*}{ Axonal injury } & $\begin{array}{l}\text { Axonal microtubule associated protein } \\
\text { (Tau) or cleaved-Tau (c-Tau) }\end{array}$ \\
\hline & Neurofilament protein-H (NF-H) \\
\hline Dendritic injury & $\begin{array}{l}\text { Dendritic microtubule associated } \\
\text { protein-2 (MAP-2) }\end{array}$ \\
\hline $\begin{array}{l}\text { Pro-inflammatory } \\
\text { cytokines }\end{array}$ & $\begin{array}{l}\text { Interleukins-1, 6, and } 8 \\
\text { (IL-1, IL-6, IL-8, TNF-alpha, IFN- } \gamma \text { ) }\end{array}$ \\
\hline $\begin{array}{l}\text { Brain specific fatty } \\
\text { acid-binding protein }\end{array}$ & B-FABP \\
\hline Gene expression & $\begin{array}{l}\text { MicroRNAs (miR-16, miR- 26a, } \\
\text { miR-92a, miR-638, and miR-765) }\end{array}$ \\
\hline
\end{tabular}

$S B D P$ spectrin breakdown product, $T N F \alpha$ tumor necrosis factor alpha, $I F N-\gamma$ interferon gamma, $B-F A B P$ brain fatty acid-binding protein cytochrome $\mathrm{C}$ release also have been correlated with the initiation of cell death in TBI [23]. Additional markers elevated during cellular injury include creatine kinase (CK) and lactate dehydrogenase (LDH), and have been extensively studied [21]. More recently, Redell and colleagues [24] investigated several microRNAs (miR-16, miR-26a, miR-92a, miR-638, and miR-765) in TBI and found consistent patterns in mild versus severe brain injury. In addition, they found an association between blast injury and a predictable appearance of microRNA Let-7i. Further investigations in molecules that regulate gene expression at the post-transcriptional level may prove valuable.

Confounding factors to the reliability of biomarkers in the geriatric population may include the influence of the aging process, presence of comorbid conditions, and/or altered clearance, distribution or elimination. For example, circulating levels of ubiquitous markers such as inflammatory cytokines IL-1, -6 , and -10 and TNF- $\alpha$ have been shown to be altered by conditions such as hypertension, diabetes, dementia, Parkinson's disease, and osteoporosis, and even by changes in body composition, all common among elderly patients $[25,26]$. It would follow that other nonspecific markers such as $\mathrm{LDH}$ and $\mathrm{CK}$ may also lack consistency in TBI.

\section{Discussion}

One of the greatest barriers to the appropriate care of geriatric patients has been identifying a means to detect and appropriately stratify degrees of neurologic injury. Due to the paucity of data regarding appropriate TBI identification and management in the aging patient, current guidelines have been derived primarily from data gathered in younger patients. Traditionally, patients have been categorized into one of three groups; mild, moderate, and severe, based on severity according to the Glasgow Coma Scale (GCS) [27]. 
The GCS offers a snapshot of gross neurologic function, but it fails to adequately identify the true underlying pathology and is subject to change as the injury progresses. In addition, GCS may be a less reliable measure of severity of injury in older individuals because of numerous factors, including sensory deterioration and pre-existing dementia [28]. A more accurate means to correlate extent of the disease with prognosis may be the Marshall head CT classification where a detailed anatomic description of injury is utilized, although its use is not widespread [29]. CT and magnetic resonance imaging may expand our understanding of intracranial anatomic derangements, but the extent of cellular injury remains elusive. Neither provides specific information about the pathophysiologic mechanisms responsible for neurologic deficits, which should ultimately be the target of medical interventions [30•]. However, the emergence of new technologies such as cerebral microdialysis, advances in brain imaging, and identification of TBI-specific biological markers may expand our ability to appropriately stratify and manage TBI. Recently, the pursuit of neuronal biomarker panels has gained momentum for the potential to provide real-time monitoring of the severity and progression of injury at the cellular level [31]. Knowledge at this level of detail may translate into injury-specific TBI stratification and titration and optimization of treatment modalities, and may guide development of cutting edge therapeutics [32].

Over the past several years, investigators have targeted potential neuronal biomarkers present in CSF and serum following TBI. It is believed that specific neuronal biomarkers may be used to detect brain ischemia, apoptosis and cellular death, in the same manner as biomarker panels are currently utilized to quantify myocardial and hepatic insult. Emerging technology may soon pave the way for the use of biochemical markers in the diagnosis, treatment and prognosis in older patients. This technology has the potential to identify a panel of biochemical markers detectable for TBI in serum and human CSF, biological fluids routinely accessible. The long-term goal is to produce quantitative enzymelinked immunosorbent assay (ELISA) systems capable of providing clinically useful diagnostic and prognostic information from both CSF and blood samples, much similar to the panel currently used to identify myocardial injury. In addition, converting such brain injury biomarker ELISAs into a blood-based point of care (POC) rapid diagnostic format (with results obtainable within 15-20 $\mathrm{min}$ ) will be another key milestone towards widespread adaptation into emergency care practice for TBI.

\section{Conclusions}

TBI remains a significant source of morbidity and mortality, and identifying optimal management strategies may contribute to improved neurologic outcome. Aggressive adherence to treatment guidelines such as those established by the Brain Trauma Foundation (BTF) has proven to reduce morbidity and mortality in the general population but none specifically address the geriatric population. To date there are no reliable predictors of neurologic dysfunction. There are several promising neuro-specific biochemical markers that may aid in the molecular diagnosis, ongoing assessment, and clinical management of these patients. Overall, it is difficult to speculate at this time which of these biomarkers will prove to be the ideal candidates in the geriatric population. The next phase of research will seek to identify the influence of the aging process and comorbid conditions on biomarker production, distribution, metabolism, and clearance, which have yet to be fully elucidated.

\section{Disclaimer}

The views expressed in this article are those of the author(s) and do not necessarily reflect the official policy or position of the Department of the Navy, Department of Defense or the United States Government.

Acknowledgements Author DR is a military service member. This work was prepared as part of official duties. Title 17 U.S.C. 105 provides that 'Copyright protection under this title is not available for any work of the United States Government.' Title 17 U.S.C. 101 defines a United States Government work as a work prepared by a military service member or employee of the United States Government as part of that person's official duties.

Disclosures CDR D. Rice USN, M.D., Ph.D. none. K.K.W. Wang, Ph.D. owns stock of Banyan Biomarkers, Inc. S. Robicsek M.D., Ph.D., none.

\section{References}

Papers of particular interest, published recently, have been highlighted as:

- Of importance

•- Of major importance

1. The Silver Book: Chronic Disease and Medical Innovation in an Aging Nation [database on the Internet]. Alliance for Aging Research. 2012. Available from: www.agingresearch.org. Accessed: 2012.

2. Langlois JA, Rutland-Brown W, Wald MM. The epidemiology and impact of traumatic brain injury: a brief overview. J Head Trauma Rehabil. 2006;21(5):375-8.

3. Dikmen SS, Machamer JE, Powell JM, Temkin NR. Outcome 3 to 5 years after moderate to severe traumatic brain injury. Arch Phys Med Rehabil. 2003;84(10):1449-57.

4. Hukkelhoven CW, Steyerberg EW, Rampen AJ, Farace E, Habbema JD, Marshall LF, et al. Patient age and outcome following severe traumatic brain injury: an analysis of 5600 patients. J Neurosurg. 2003;99(4):666-73. 
5. Percent Distribution of the Projected Population by Selected Age Groups and Sex for the United States: 2010 to 2050. In: Population Division USCB, editor.: U.S. Census Bureau; 2008. http:// www.census.gov/population/www/projections/files/nation/summary/ np2008-t3.xls

6. Thompson HJ, McCormick WC, Kagan SH. Traumatic brain injury in older adults: epidemiology, outcomes, and future implications. J Am Geriatr Soc. 2006;54(10):1590-5.

7. Mosenthal AC, Lavery RF, Addis M, Kaul S, Ross S, Marburger R, et al. Isolated traumatic brain injury: age is an independent predictor of mortality and early outcome. J Trauma. 2002;52(5):907-11.

8. Jiang JY, Gao GY, Li WP, Yu MK, Zhu C. Early indicators of prognosis in 846 cases of severe traumatic brain injury. J Neurotrauma. 2002;19(7):869-74.

9. • Papa L, Akinyi L, Liu MC, Pineda JA, Tepas JJ, 3rd, Oli MW, et al. Ubiquitin C-terminal hydrolase is a novel biomarker in humans for severe traumatic brain injury. Crit Care Med. ;38(1):138-44. Novel neuronal biomarker UCH-L1 as disanotics for severe TBI.

10. Coronado VG, Thomas KE, Sattin RW, Johnson RL. The CDC traumatic brain injury surveillance system: characteristics of persons aged 65 years and older hospitalized with a TBI. J Head Trauma Rehabil. 2005;20(3):215-28.

11. - Koskinen S, Alaranta H. Traumatic brain injury in Finland 1991-2005: a nationwide register study of hospitalized and fatal TBI. Brain Inj. 2008;22(3):205-14. Comprehensive register of severe TBI population.

12. Sahuquillo J, Poca M, Amorós S. Current aspects of pathophysiology and cell dysfunction after severe head injury. Curr Pharm Des. 2001;7:1475-503.

13. Povlishock JT, Katz DI. Update of neuropathology and neurological recovery after traumatic brain injury. J Head Trauma Rehabil. 2005;20(1):76-94.

14. Kass I. Handbook of neuroanesthesia. 4th ed. Philadelphia: Lippincott, Williams, and Wilkins; 2007.

15. Maas AI, Stocchetti N, Bullock R. Moderate and severe traumatic brain injury in adults. Lancet Neurol. 2008;7(8):728-41.

16. Morganti-Kossmann MC, Satgunaseelan L, Bye N, Kossmann T. Modulation of immune response by head injury. Injury. 2007;38:1392-400.

17. Pitts L, McIntosh T. Head Injury. In: Braakman R, editor. Handbook of clinical neurology. Amsterdam: Elsevier Science Publishers; 1990 . p. $65-100$.

18. Gilmer LK, Ansari MA, Roberts KN, Scheff SW. Age-related mitochondrial changes after traumatic brain injury. J Neurotrauma. 2010;27(5):939-50.

19. Kochanek PM, Berger RP, Bayir H, Wagner AK, Jenkins LW, Clark RS. Biomarkers of primary and evolving damage in traumatic and ischemic brain injury: diagnosis, prognosis, probing mechanisms, and therapeutic decision making. Curr Opin Crit Care. 2008;14(2):135-41.

20. Ottens AK, Golden EC, Bustamante L, Hayes RL, Denslow ND, Wang KK. Proteolysis of multiple myelin basic protein isoforms after neurotrauma: characterization by mass spectrometry. J Neurochem. 2008;104(5):1404-14.

21. Pineda JA, Wang KK, Hayes RL. Biomarkers of proteolytic damage following traumatic brain injury. Brain Pathol. 2004;14(2):202-9.

22. Zhang Z, Larner SF, Liu MC, Zheng W, Hayes RL, Wang KK. Multiple alphaII-spectrin breakdown products distinguish calpain and caspase dominated necrotic and apoptotic cell death pathways. Apoptosis. 2009;14(11):1289-98.

23. Zhao H, Yenari MA, Cheng D, Sapolsky RM, Steinberg GK. Biphasic cytochrome c release after transient global ischemia and its inhibition by hypothermia. J Cereb Blood Flow Metab. 2005;25(9):1119-29.

24. Balakathiresan N, Bhomia M, Chandran R, Chavko M, McCarron RM, Maheshwari RK. MicroRNA let-7i is a promising serum biomarker for blast-induced traumatic brain injury. J Neurotrauma. 2012;29(7):1379-87.
25. Krabbe KS, Pedersen M, Bruunsgaard H. Inflammatory mediators in the elderly. Exp Gerontol. 2004;39(5):687-99.

26. Bruunsgaard H, Pedersen BK. Age-related inflammatory cytokines and disease. Immunol Allergy Clin North Am. 2003;23(1):15-39.

27. Teasdale G, Jennett B. Assessment of coma and impaired consciousness. A practical scale. Lancet. 1974;2(7872):81-4.

28. Powers R. Neurobiology of Aging. In: Coffey C, Cummings JL, editors. Textbook of geriatric neuropsychiatry. Washington: American Psychiatric Press; 2000. p. 33-79.

29. Marshall LF, Marshall SB, Klauber MR, Van Berkum Clark M, Eisenberg H, Jane JA, et al. The diagnosis of head injury requires a classification based on computed axial tomography. J Neurotrauma. 1992;9 Suppl 1:S287-92.

30. - Saatman KE, Duhaime AC, Bullock R, Maas AI, Valadka A, Manley GT. Classification of traumatic brain injury for targeted therapies. J Neurotrauma. 2008;25(7):719-38. This is an imporant review article covering the contemporary classification of TBI.

31. Kobeissy FH, Ottens AK, Zhang Z, Liu MC, Denslow ND, Dave $\mathrm{JR}$, et al. Novel differential neuroproteomics analysis of traumatic brain injury in rats. Mol Cell Proteomics. 2006;5(10):1887-98.

32. Zhang Z, Mondello S, Kobeissy FH, Rubenstein R, Streeter J, Hayes $\mathrm{RL}$, et al. Protein biomarkers for traumatic and ischemic brain injury: from bench to bedside. Transl Stroke Res. 2011;2:455-62. review.

33. Pelinka LE, Kroepfl A, Leixnering M, Buchinger W, Raabe A, Redl H. GFAP versus S100B in serum after traumatic brain injury: relationship to brain damage and outcome. J Neurotrauma. 2004;21 (11):1553-61.

34. Vos PE, Lamers KJ, Hendriks JC, van Haaren M, Beems T, Zimmerman $\mathrm{C}$, et al. Glial and neuronal proteins in serum predict outcome after severe traumatic brain injury. Neurology. 2004;62(8):1303-10.

35. Zemlan FP, Jauch EC, Mulchahey JJ, Gabbita SP, Rosenberg WS, Speciale SG, et al. C-tau biomarker of neuronal damage in severe brain injured patients: association with elevated intracranial pressure and clinical outcome. Brain Res. 2002;947(1):131-9.

36. Siman R, Toraskar N, Dang A, McNeil E, McGarvey M, Plaum J, et al. A panel of neuron-enriched proteins as markers for traumatic brain injury in humans. J Neurotrauma. 2009;26(11):1867-77.

37. Petzold A. Neurofilament phosphoforms: surrogate markers for axonal injury, degeneration and loss. J Neurol Sci. 2005;233(12):183-98.

38. Petzold A, Shaw G. Comparison of two ELISA methods for measuring levels of the phosphorylated neurofilament heavy chain. J Immunol Methods. 2007;319(1-2):34-40.

39. Park D, Joo SS, Lee HJ, Choi KC, Kim SU, Kim YB. Microtubuleassociated protein 2, an early blood marker of ischemic brain injury. J Neurosci Res. 2012;90(2):461-7.

40. •• Papa L, Lewis L, Falk J, Silvestri S, Giordano P, Brophy GM, et al. Serum levels of ubiquitin C-terminal hydrolase (UCH-L1) distinguish mild traumatic brain injury from trauma controls and are elevated in mild and moderate tbi patients with intracranial lesions. J Trauma. 2012;72:1335-44. This study shows that a new neuronal marker UCH-L1 can make its way to circulating blood even after mild to moderate TBI.

41. • Papa L, Lewis L, Falk J, Zhang Z, Silvestri S, Giordano P, et al. Glial fibrillary acidic protein breakdown product as a novel serum biomarker for mild and moderate traumatic brain injury. Ann Emerg Med. 2012;59(6):471-83. This study shows that a glial origin marker can make its way to circulating blood even after mild to moderate TBI.

42. Maier B, Laurer HL, Rose S, Buurman WA, Marzi I. Physiological levels of pro- and anti-inflammatory mediators in cerebrospinal fluid and plasma: a normative study. J Neurotrauma. 2005;22(7):822-35.

43. Chiaretti A, Antonelli A, Mastrangelo A, Pezzotti P, Tortorolo L, Tosi F, et al. Interleukin-6 and nerve growth factor upregulation correlates with improved outcome in children with severe traumatic brain injury. J Neurotrauma. 2008;25(3):225-34. 